\title{
Parallel Decentralized Network for the Detection of Unknown Signals Through Wireless Nakagami Fading Channels
}

\author{
Ebtehal H. El-Bahaie ${ }^{1}$ and Emad K. Al-Hussaini ${ }^{2}$ \\ ${ }^{1}$ Electronics and Communications Engineering Department, Misr International University, Cairo, Egypt \\ ${ }^{2}$ Electronics and Communications Department, Faculty of Engineering, Cairo University, 12613 Giza, Egypt
}

Correspondence should be addressed to Ebtehal H. El-Bahaie, ebtehal.elbahaie@gmail.com

Received 15 October 2009; Revised 25 February 2010; Accepted 24 April 2010

Academic Editor: George S. Tombras

Copyright ( 2010 E. H. El-Bahaie and E. K. Al-Hussaini. This is an open access article distributed under the Creative Commons Attribution License, which permits unrestricted use, distribution, and reproduction in any medium, provided the original work is properly cited.

\begin{abstract}
In this paper analytical and simulation results for the decentralized detection of unknown signals are introduced. Parallel sensor network scheme is assumed. Additive White Gaussian Noise (AWGN) and Nakagami fading are assumed in both links, that is, from the source to the decentralized local sensors and from the sensors to the fusion center. Furthermore, diversity employing Square Law Combining (SLC) or Square Law Selection (SLS) is considered at each sensor, for both independent and correlated branches. Appreciable improvements are obtained with the increase of the number of sensors and the diversity employment.
\end{abstract}

\section{Introduction}

In past researches [1-5], signal processing with distributed sensors had gained importance due to the relatively low cost of sensors, the inherent redundancy possible with multiple sensors, the availability of high speed communication links, and increased computational capability. Decentralized detection using a group of local sensors to detect the presence or absence of a certain target (phenomenaunknown signal) was originally used mainly in military tracking, radar systems, and control applications but now is being employed in a wide variety of applications including scientific, industrial, health care, agriculture, and domestic applications. In decentralized processing, some preliminary processing is carried out at each sensor, and the information is then sent from each sensor to a central processor which is known as the fusion center. In previously reported work the processing at the detectors and at the fusion center was optimized. Chair and Varshney [3] derived the optimum fusion rule at the data fusion center for a Bayesian detection problem with distributed sensors. Lee and Chao [4] presented a distributed detection scheme based on soft local decisions. In [5] nonparametric tests such as the Sign and Wilcoxon detectors were considered for the situations when the signal to be detected is embedded in a noise whose characteristics are not completely known. In [6], Niu, Chen and Varshney presented a model for the decisions made by the local sensors and transmitted to the fusion center through noisy and Rayleigh fading channels. This paper includes analysis of the system in two stages. The first stage consists of the detection of an unknown signal transmitted through Nakagami fading channels using a group of local sensors that employ an energy detection technique. The energy in the received waveform is measured over an observation window. The problem of detecting an unknown deterministic signal over a Gaussian noise channel by employing the energy detection technique was first addressed by Urkowitz [7]. It was revisited in [8] for Rayleigh and Nakagami fading channels assumed in the detection problem. In [8], single stage was assumed, no decentralized network was considered, and no results were obtained for noninteger Nakagami fading parameter. In this paper, expressions for probabilities of false-alarm and detection at each local sensor are derived under the presence of AWGN and Nakagami fading for general fading parameters. Furthermore, in the second stage, decisions made by the local sensors are transmitted through Nakagami fading and AWGN channels to a fusion center where expressions for the probabilities of false alarm 
and detection are obtained. The choice of the Nakagami fading is due to its flexibility in matching experimental data rather than the use of Rayleigh, log-normal, or Rice distributions. Rayleigh distribution represents a special case from it. Furthermore, it can fit less and severe fading than that of Rayleigh. This paper is organized as follows. The system model is described in Section 2. In Section 3, the false alarm and detection probabilities over AWGN and Nakagami fading channels are derived at the local sensors and the fusion center, respectively. Diversity using Square Law Combining (SLC) and Square Law Selection (SLS) schemes are employed at the local sensors in Section 4, and the probability of detection in both cases is also evaluated. Finally, numerical and simulation results are demonstrated in Section 5, and the conclusion is offered in Section 6.

\section{System Model}

The system under consideration is shown in Figure 1. As shown in figure, parallel sensor network scheme is assumed. Parallel data vectors are transmitted to each sensor through independent wireless noisy fading channels in the first stage. Each sensor makes its own binary decision. These decisions are transmitted in the second stage through wireless noisy fading channels to a fusion center where a global decision is obtained.

The system parameters are defined as follows.

$h_{i j}$ : Nakagami fading channel for sensor $i$ of stage $j$,

$n_{i j}(t)$ : noise waveform modeled as zero mean Additive White Gaussian Noise at sensor $i$ and stage $j$,

$P_{d i j}$ : Probability of detection at sensor $i$ and stage $j$,

$P_{f i j}$ : Probability of false alarm at sensor $i$, where $1 \leq i \leq K$, where $K$ is the number of sensors, and $j=1,2$ refers to the first and second stage, respectively,

$u_{i}$ : decision made by local sensor $i$,

$P_{D}$ : Probability of detection at the Fusion Center,

$P_{F}$ : Probability of false alarm at the Fusion Center.

The signal to be detected is assumed to be unknown and occurs on the basis of two hypotheses $H_{1}$ and $H_{0}$ indicating signal presence or absence, respectively. Our analysis applies to either low-pass (LP) or band-pass (BP) system, however we focus on BP representation. The received signal at local sensor $i$ can be represented as

$$
r_{i}(t)= \begin{cases}\mathfrak{R}\left\{\left[h_{i j} S_{i}(t)+n_{i j}(t)\right] e^{-j 2 \pi f_{c} t}\right\}, & H_{1}, \\ \Re\left\{n_{i j}(t) e^{-j 2 \pi f_{c} t}\right\}, & H_{0},\end{cases}
$$

where $\mathfrak{R}\{\cdot\}$ denotes the real operation, $f_{c}$ is the carrier frequency.

$S_{i}(t)$ is an equivalent LP representation of the unknown signal at sensor $i$ with bandwidth $W$. Let $S_{c}(t)$ and $S_{s}(t)$ denote the inphase $(I)$ and quadrature $(Q)$ components, respectively, that is,

$$
S_{i}(t)=S_{c}(t)+j S_{s}(t)
$$

Similarly the AWGN $n_{i}(t)$ with one sided PSD $N_{0}$ can be written as

$$
n_{i}(t)=n_{c}(t)+j n_{s}(t) .
$$

Therefore, the $I$ and $Q$ components will be each confined to the frequency support $[-W / 2, W / 2]$. Finally, let $\gamma=$ $\alpha_{i}^{2} E_{\mathrm{s}} / N_{0}$ denotes the SNR, where $E_{\mathrm{s}}$ is the signal energy, and $\alpha$ represents the fading amplitude which is assumed to follow a Nakagami distribution. Thus, the Probability Density Function (PDF) of $\gamma$ over a Nakagami channel can be written as follows [8, Equation (6)]

$$
f_{\mathrm{Nak}}(\gamma)=\frac{1}{\Gamma(m)}\left(\frac{m}{\bar{\gamma}}\right)^{m} \gamma^{m-1} \exp \left(-\frac{m \gamma}{\bar{\gamma}}\right), \quad \gamma \geq 0,
$$

where $m$ is the Nakagami parameter, and $\bar{\gamma}$ is the average SNR.

The receiver structure implemented by the local sensor can generally be described as follows. The received signal is first prefiltered by an ideal BP filter. Then, the output of this filter is squared and integrated over a time interval $T$ to finally produce a measure of the energy of the received waveform. The output of the integrator denoted by $g$ can be expressed as

$g_{i}$

$$
\begin{aligned}
& \triangleq \frac{2}{N_{0}} \int_{0}^{T} r_{i}{ }^{2}(t) d t \\
& \cong \frac{1}{N_{0} W}\left[\sum_{i=1}^{N / 2}\left(\alpha_{c} S_{c i}-\alpha_{s} S_{s i}+n_{c i}\right)^{2}+\sum_{i=1}^{N / 2}\left(\alpha_{c} S_{s i}+\alpha_{s} S_{c i}+n_{s i}\right)^{2}\right],
\end{aligned}
$$

where $N / 2$ is the number of samples per either $I$ or $Q$ components, $\alpha_{c}=\alpha \cos \theta, \alpha_{s}=\alpha \sin \theta$, and generally $x_{c k}$ and $x_{s k}$, respectively, denote the $k$ th samples of $x_{c}(t)$ and $x_{s}(t)$, that is, $I$ and $Q$ components. It then follows that under $H_{1}, g$ has a noncentral chi-square distribution with variance $\sigma^{2}$, non centrality parameter $\mu=2 \gamma$ and $N$ Degrees of Freedom (DOFs). Under $H_{0}, g$ has central chi-square distribution. Throughout the following analysis, we will consider $\mu=a \gamma$ and take the special case of $a=2$ and $\sigma^{2}=1$. Thus the PDF of $g$ is given by [8, Equation (3)]

$$
\begin{aligned}
& f_{G}(g) \\
& = \begin{cases}\frac{1}{\sigma^{N} 2^{N / 2} \Gamma(N / 2)} g^{(N / 2)-1} e^{-g / 2 \sigma^{2}}, & H_{0}, \\
\frac{1}{2 \sigma^{2}}\left(\frac{g}{a \gamma}\right)^{(N-2) / 4} e^{-(a \gamma+g) / 2 \sigma^{2}} I_{(N / 2)-1}\left(\frac{\sqrt{a \gamma g}}{\sigma^{2}}\right), & H_{1},\end{cases}
\end{aligned}
$$

where $\Gamma(\cdot)$ is the gamma function and $I_{v}(\cdot)$ is the $v$ th-order modified Bessel function of the first kind [9, 2.1-120].

\section{Analytical Results}

In the following Sections 3.1 and 3.2, false alarm and detection probabilities are derived for the first and second stage, respectively. 


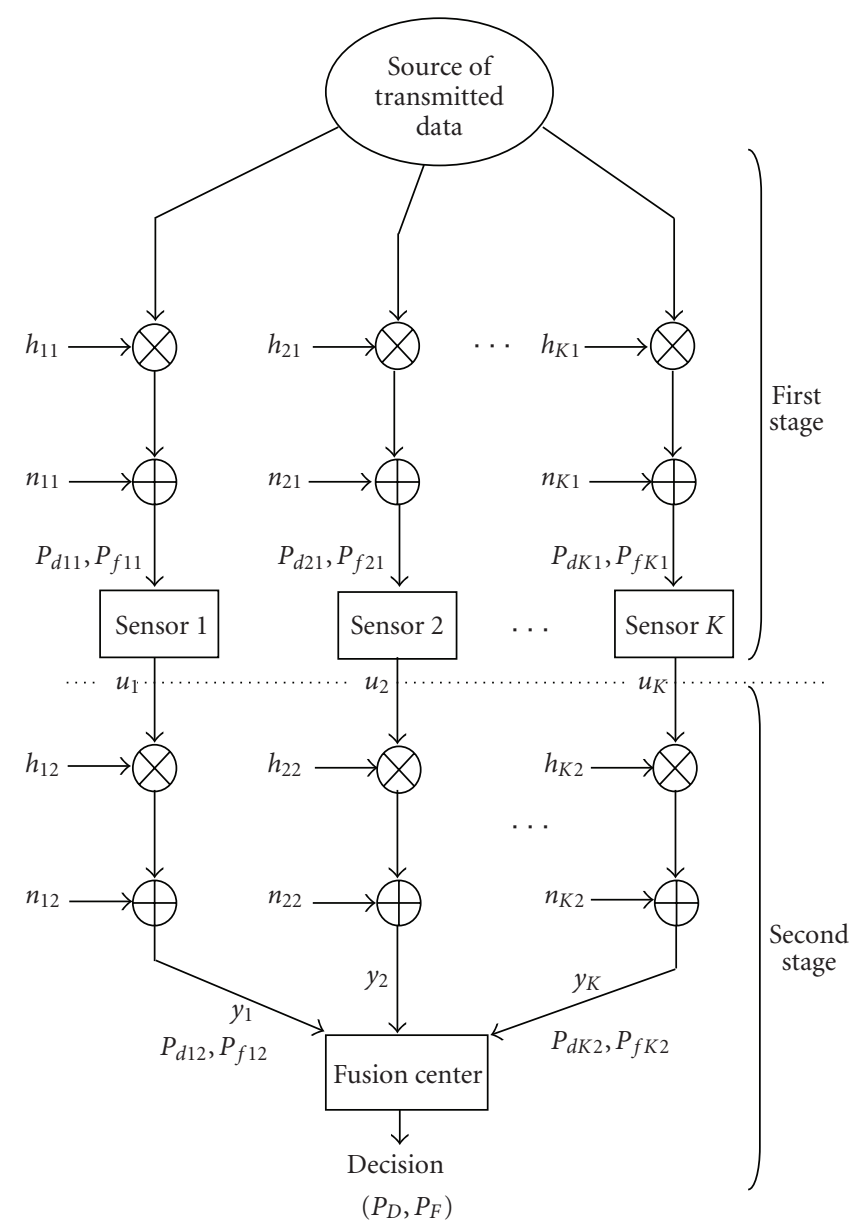

Figure 1: The two stage system model.

3.1. False Alarm and Detection Probabilities at the Local Sensors. In this section, we define $P_{f_{i 1}}=\operatorname{Pr}\left(g_{i}>\lambda \mid H_{0}\right)$ and $P_{d_{i 1}}=\operatorname{Pr}\left(g_{i}>\lambda \mid H_{1}\right)$ as the probability of false alarm and the probability of detection for sensor $i$, respectively, where $\lambda$ is a decision threshold. The local sensors are assumed to be identical, thus for simplicity, $P_{f_{i 1}}=P_{f_{1}}$ and $P_{d_{i 1}}=P_{d_{1}}$. Based on the statistics of $g, P_{f_{1}}$ over AWGN channels can be written as $[8$, equation (4)]

$$
P_{f_{1}}=\frac{\Gamma\left(N / 2, \lambda / 2 \sigma^{2}\right)}{\Gamma(N / 2)}
$$

where $\Gamma(\cdot, \cdot)$ is the incomplete gamma function. Similarly $P_{d_{1}}$ is given by [8, equation (5)]

$$
P_{d_{1}}=Q_{N / 2}\left(\sqrt{\frac{a \gamma}{\sigma^{2}}}, \sqrt{\frac{\lambda}{\sigma^{2}}}\right),
$$

where $Q_{N / 2}(\cdot, \cdot)$ is the generalized Marcum $Q$-function [10, equation (4.59)].
By averaging (8) over (4), and with the aid of Appendix A.1, the average $P_{d_{1}}$ over a Nakagami channel, $P_{d 1, \text { Nak }}$, can be obtained as

$$
\begin{aligned}
P_{d 1, \mathrm{Nak}}=\zeta G_{1} & +\left(\frac{2 m \sigma^{2}}{2 m \sigma^{2}+a \bar{\gamma}}\right)^{m} e^{-\lambda / 2 \sigma^{2}} \sum_{i=1}^{(N / 2)-1} \frac{\left(\lambda / 2 \sigma^{2}\right)^{i}}{i !} \\
& \times{ }_{1} F_{1}\left(m ; i+1 ; \frac{\lambda a \bar{\gamma}}{2 \sigma^{2}\left(2 m \sigma^{2}+a \bar{\gamma}\right)}\right),
\end{aligned}
$$

where $\zeta=2 / \Gamma(m)\left(m \sigma^{2} / a \bar{\gamma}\right)^{m}$, and ${ }_{1} F_{1}(\cdot ; \cdot ; \cdot)$ is the confluent hyper-geometric function [11, 9.21]. For integer $m, G_{1}$ is given by

$$
\begin{aligned}
G_{1}= & \frac{2^{m-1}(m-1) !}{v^{2 m}} \frac{b_{1}{ }^{2}}{v^{2}+b_{1}{ }^{2}} \exp \left(-\frac{b_{2}{ }^{2}}{2} \frac{v^{2}}{v^{2}+b_{1}^{2}}\right) \\
& \times \sum_{k=0}^{m-1} \epsilon_{k}\left(\frac{v^{2}}{v^{2}+b_{1}^{2}}\right)^{k} L_{k}\left(-\frac{b_{2}^{2}}{2} \frac{b_{1}^{2}}{v^{2}+b_{1}^{2}}\right),
\end{aligned}
$$


where $L_{k}$ is the laguerre polynomial of degree $k[11,8.970]$, and

$$
\epsilon_{k}= \begin{cases}1, & k<m-1, \\ 1+\frac{v^{2}}{b_{1}^{2}}, & k=m-1 .\end{cases}
$$

Whereas for the special case of $m=1 / 2$ which represents the worst fading condition of the Nakagami distribution, $G_{1}$ is given by

$$
\begin{aligned}
G_{1}=\sqrt{\frac{\pi}{2} \frac{1}{v}}\left[2 Q_{1}\left(\frac{b_{2}}{2}\left(1-\frac{v}{s}\right), \frac{b_{2}}{2}\left(1+\frac{v}{s}\right)\right)\right. \\
\left.\quad-\exp \left(-\frac{b_{1}^{2}+2 v^{2}}{s^{2}} \frac{b_{2}{ }^{2}}{4}\right) I_{0}\left(\frac{b_{1}{ }^{2} b_{2}{ }^{2}}{4 s^{2}}\right)\right],
\end{aligned}
$$

where $v^{2}=2 m \sigma^{2} / a \bar{\gamma}, b_{1}=1, b_{2}=\sqrt{\lambda / \sigma^{2}}, s=\sqrt{b_{1}^{2}+v^{2}}$, and $I_{0}(\cdot)$ is the modified Bessel function of order zero.

Notice that $P_{f_{1}}$ in case of fading channels will remain as in (7), since it is independent on the SNR.

\subsection{False Alarm and Detection Probabilities at the Fusion} Center. As shown in Figure 1, the second stage consists of the binary decisions $u_{i} \in\{+1,-1\}, i=1,2, \ldots, K$ made by the local sensors and transmitted to the fusion center through fading and noisy channels and $u_{i}=+1$ or -1 under $H_{1}$ and $H_{0}$, respectively. The detection performance of each local sensor can be characterized by its corresponding probability of false alarm and detection denoted by $P_{f_{1}}$ and $P_{d 1, \mathrm{Nak}}$, respectively, that were defined in Section 3.1.

The output of the channel (input to the Fusion center) for the ith sensor is defined as

$$
y_{i}=x_{i}+n_{i}=h_{i} u_{i}+n_{i},
$$

where $h_{i}$ is Nakagami fading with PDF given by [12, equation (1)]

$$
f\left(h_{i}\right)=\frac{2 m^{m} h_{i}^{2 m-1}}{\Gamma(m) \Omega^{m}} e^{\left(-m h_{i}{ }^{2} / \Omega\right)}, \quad h_{i}>0 .
$$

In the following analysis $\Omega=E\left(h_{i}{ }^{2}\right)$ is normalized. It is to be noted that (14) is equivalent to (4) except that the $\operatorname{SNR}(\gamma)$ is replaced by the amplitude $h_{i}$.

To obtain an expression for the probability of detection at the output of each channel (input to fusion center) it is necessary to find $p\left(y_{i} \mid H_{1}\right)$ and $p\left(y_{i} \mid H_{0}\right)$.

Let us now consider $p\left(y_{i} \mid H_{1}\right)$. The first step is to find $p\left(u_{i} \mid H_{1}\right)$ :

$$
p\left(u_{i} \mid H_{1}\right)= \begin{cases}P_{d 1, \mathrm{Nak}}, & u_{i}=1, \\ 1-P_{d 1, \mathrm{Nak}}, & u_{i}=-1 .\end{cases}
$$

From (13), $f\left(x_{i} \mid u_{i}\right)$ can be written as

$$
f\left(x_{i} \mid u_{i}\right)=\frac{2 m^{m}}{\Gamma(m)} u_{i} x_{i}^{2 m-1} \exp \left(-m x_{i}^{2}\right) U\left(u_{i} x_{i}\right)
$$

where $U(\cdot)$ is a step function defined as the following:

$$
U(x)= \begin{cases}1, & x \geq 0 \\ 0, & x<0 .\end{cases}
$$

Because $n_{i}$ is Gaussian distributed with zero mean and variance $\sigma_{n}{ }^{2}, f\left(y_{i} \mid x_{i}\right)$ is given by

$$
f\left(y_{i} \mid x_{i}\right)=\frac{1}{\sqrt{2 \pi} \sigma_{n}} e^{-\left(y_{i}-x_{i}\right)^{2} / 2 \sigma_{n}{ }^{2}} .
$$

Thus, the following result for $f\left(y_{i} \mid x_{i}\right)$ is obtained as follows:

$$
\begin{aligned}
f\left(y_{i} \mid u_{i}\right) & =\int f\left(x_{i} \mid u_{i}\right) f\left(y_{i} \mid x_{i}, u_{i}\right) d x_{i} \\
& =\int f\left(x_{i} \mid u_{i}\right) f\left(y_{i} \mid x_{i}\right) d x_{i},
\end{aligned}
$$

where the identity $f\left(y_{i} \mid x_{i}, u_{i}\right)=f\left(y_{i} \mid x_{i}\right)$, which is obtained from the fact that $u_{i}, x_{i}$, and $y_{i}$ form a Markov chain, has been used.

By substituting (16) and (18) into (19) and setting $u_{i}=$ 1 , a general integral form, that can be used to evaluate the conditional PDF $f\left(y_{i} \mid u_{i}\right)$ for any Nakagami parameter " $m$ ", can be written as

$$
\begin{aligned}
& f\left(y_{i} \mid u_{i}=1\right) \\
& \quad=\beta \int_{0}^{\infty} x^{2 m-1} \exp \left(-\frac{\left(x-\left(y /\left(2 m \sigma_{n}^{2}+1\right)\right)\right)^{2}}{2 \sigma_{n}^{2} /\left(2 m \sigma_{n}^{2}+1\right)}\right) d x .
\end{aligned}
$$

Similarly for $u_{i}=-1$,

$$
\begin{aligned}
f\left(y_{i} \mid\right. & \left.u_{i}=-1\right) \\
& =\beta \int_{0}^{\infty} x^{2 m-1} \exp \left(-\frac{\left(x+\left(y /\left(2 m \sigma_{n}{ }^{2}+1\right)\right)\right)^{2}}{2 \sigma_{n}{ }^{2} /\left(2 m \sigma_{n}{ }^{2}+1\right)}\right) d x,
\end{aligned}
$$

where $\beta=2 m^{m} /\left(\Gamma(m) \sqrt{2 \pi} \sigma_{n}\right) e^{-m y^{2} /\left(2 m \sigma_{n}{ }^{2}+1\right)}$

In the following Sections 3.2.1, 3.2.2, and 3.2.3, three cases will be considered depending on the fading depth. In particular $m=1 / 2,1$, and 2, respectively, to obtain the probabilities of false alarm and detection at the fusion center due to each sensor independently. In Section 3.3 the overall probabilities of detection and false alarm at the fusion center are derived.

3.2.1. Case $m=1 / 2$ (worst case of Nakagami fading). Substituting $m=1 / 2$ in (20) the conditional PDF $f_{\mathrm{Nak}, 1 / 2}\left(y_{i} \mid\right.$ $u_{i}$ ) can be derived as (see Appendix B.1)

$$
\begin{aligned}
& f_{\mathrm{Nak}, 1 / 2}\left(y_{i} \mid u_{i}\right) \\
& =\frac{\sqrt{2}}{\Gamma(1 / 2) \sqrt{\sigma_{n}^{2}+1}} e^{-y_{i}{ }^{2} / 2\left(\sigma_{n}{ }^{2}+1\right)} Q\left(\frac{-u_{i} y_{i}}{\sigma_{n} \sqrt{\sigma_{n}^{2}+1}}\right),
\end{aligned}
$$

where $Q(\cdot)$ is the complementary distributed function of the standard Gaussian, that is,

$$
Q(x)=\int_{x}^{\infty} \frac{1}{\sqrt{2 \pi}} e^{-t^{2} / 2} d t .
$$


Let $f\left(y_{i} \mid H_{1}\right)$ and $f\left(y_{i} \mid H_{0}\right)$ be defined for all $m$ as in (24) and (25), respectively,

$$
\begin{aligned}
f( & \left.y_{i} \mid H_{1}\right) \\
& =\sum_{u_{i}}\left[p\left(u_{i} \mid H_{1}\right) f\left(y_{i} \mid u_{i}\right)\right] \\
& =P_{d 1, \mathrm{Nak}} f\left(y_{i} \mid u_{i}=1\right)+\left(1-P_{d 1, \mathrm{Nak}}\right) f\left(y_{i} \mid u_{i}=-1\right),
\end{aligned}
$$

$$
\begin{aligned}
f( & \left.y_{i} \mid H_{0}\right) \\
& =\sum_{u_{i}}\left[p\left(u_{i} \mid H_{0}\right) f\left(y_{i} \mid u_{i}\right)\right] \\
& =P_{f_{1}} f\left(y_{i} \mid u_{i}=1\right)+\left(1-P_{f_{1}}\right) f\left(y_{i} \mid u_{i}=-1\right),
\end{aligned}
$$

where $P_{d 1, \text { Nak }}$, is the average probability of detection given by (9) after setting $m=1 / 2$ and evaluating. From (22) and (24), $f_{\mathrm{Nak}, 1 / 2}\left(y_{i} \mid H_{1}\right)$ can be obtained as

$$
\begin{aligned}
f_{\mathrm{Nak}, 1 / 2}\left(y_{i} \mid H_{1}\right) \\
=\frac{\sqrt{2}}{\Gamma(1 / 2) \sqrt{\sigma_{n}{ }^{2}+1}} e^{-y_{i}{ }^{2} / 2\left(\sigma_{n}{ }^{2}+1\right)} \\
\quad \times\left[\left(1-2 P_{d 1, \mathrm{Nak}}\right) Q\left(\frac{y_{i}}{\sigma_{n} \sqrt{\sigma_{n}{ }^{2}+1}}\right)+P_{d 1, \mathrm{Nak}}\right] .
\end{aligned}
$$

The expression for $f_{\mathrm{Nak}, 1 / 2}\left(y_{i} \mid H_{0}\right)$ is similar to that in (26) while replacing $P_{d 1, \text { Nak }}$ by $P_{f_{1}}$. Finally, the probability of false alarm and the probability of detection at the output of each channel of the second stage can be defined as $P_{f_{2}}=p\left(y_{i} \geq\right.$ $\left.0 \mid H_{0}\right)$, and $P_{d 2, \mathrm{Nak}}=p\left(y_{i} \geq 0 \mid H_{1}\right)$ under hypotheses $H_{0}$ and $H_{1}$, respectively. $P_{d 2, \mathrm{Nak}}$ for $m=1 / 2$ is denoted by $P_{d 2, \mathrm{Nak}, 1 / 2}$ and evaluated with the aid of Appendix B.2 as follows:

$$
\begin{aligned}
& P_{d 2, \mathrm{Nak}, 1 / 2} \\
& =\int_{0}^{\infty} f_{\mathrm{Nak}, 1 / 2}\left(y_{i} \mid H_{1}\right) d y_{i} \\
& =\frac{1}{\Gamma(1 / 2)}\left[\left(1-2 P_{d 1, \mathrm{Nak}, 1 / 2)} \frac{\sigma_{n}}{2 \sqrt{\pi}} e^{\left(\sigma_{n}{ }^{2}+1\right) / 4} K_{0}\left(\frac{\sigma_{n}{ }^{2}+1}{4}\right)\right.\right. \\
& \left.\quad+P_{d 1, \mathrm{Nak}, 1 / 2} \sqrt{\pi}\right],
\end{aligned}
$$

where $K_{0}(\cdot)$ is the Bessel Function of imaginary argument as given by $[11,3.754 .2] . P_{f 2, \mathrm{Nak}, 1 / 2}$ will have a similar expression as in (27) except for plugging $P_{f_{1}}$ instead of $P_{d 1, \mathrm{Nak}, 1 / 2}$.

3.2.2. Case $m=1$ (Rayleigh fading). Following the same procedure as in Section 3.2.1 and by substituting $m=1$ in
(20), $f\left(y_{i} \mid u_{i}\right)$ for the Rayleigh fading case can be derived as (see Appendix B.3)

$$
\begin{aligned}
& f_{\text {Ray }}\left(y_{i} \mid u_{i}\right) \\
& =\frac{2 \sigma_{n}}{\sqrt{2 \pi}\left(2 \sigma_{n}^{2}+1\right)} e^{-y_{i}^{2} / 2 \sigma_{n}{ }^{2}} \\
& \quad \times\left[1+u_{i} \sqrt{2 \pi} p y_{i} e^{\left(p y_{i}\right)^{2} / 2} Q\left(-p u_{i} y_{i}\right)\right] .
\end{aligned}
$$

Using (28) and (24), as in the previous section $f_{\text {Ray }}\left(y_{i} \mid H_{1}\right)$ can be written as

$$
\begin{aligned}
& f_{\text {Ray }}\left(y_{i} \mid H_{1}\right) \\
& =\frac{2 \sigma_{n}}{\sqrt{2 \pi}\left(2 \sigma_{n}{ }^{2}+1\right)} e^{-y_{i}{ }^{2} / 2 \sigma_{n}{ }^{2}} \\
& \quad \times\left\{1+\left[P_{d 1, \text { Ray }}-Q\left(p y_{i}\right)\right] \sqrt{2 \pi} p y_{i} e^{\left(p y_{i}\right)^{2} / 2}\right\},
\end{aligned}
$$

where $p=1 / \sigma_{n} \sqrt{2 \sigma_{n}^{2}+1}$, and $P_{d 1 \text {, Ray }}$ is the value of $P_{d 1, \text { Nak }}$ in (9) while substituting $m=1$. Similarly, $f_{\text {Ray }}\left(y_{i} \mid H_{0}\right)$ is obtained with the same form of $f_{\text {Ray }}\left(y_{i} \mid H_{1}\right)$ as in (29) while replacing $P_{d 1 \text {, Ray }}$ with $P_{f_{1}}$.

Moreover, $P_{d 2 \text {,Ray }}$ and $P_{f 2 \text {,Ray }}$ can be obtained as in [6, equations (20) and (21)]:

$$
\begin{aligned}
& P_{f 2, \text { Ray }}=\frac{1}{2}+\frac{P_{f_{1}}-1 / 2}{\sqrt{2 \sigma_{n}^{2}+1}}, \\
& P_{d 2, \text { Ray }}=\frac{1}{2}+\frac{P_{d 1, \text { Ray }}-1 / 2}{\sqrt{2 \sigma_{n}^{2}+1}} .
\end{aligned}
$$

3.2.3. Case $m=2$ (less fading than that of Rayleigh). For Nakagami $m=2, f_{\mathrm{Nak}, 2}\left(y_{i} \mid u_{i}\right)$ can be obtained, with the aid of Appendix B.4, as follows:

$$
\begin{aligned}
& f_{\mathrm{Nak}, 2}\left(y_{i} \mid u_{i}\right) \\
& =\frac{8}{\sqrt{2 \pi} \sigma_{n}\left(4 \sigma_{n}{ }^{2}+1\right)^{3}} e^{-y_{i}{ }^{2} / 2 \sigma_{n}{ }^{2}} \\
& \quad \times\left\{y_{i}{ }^{2}{\sigma_{n}}^{2}+2 \sigma_{n}{ }^{4}\left(4 \sigma_{n}{ }^{2}+1\right)+u_{i} Q\left(-u_{i} q y_{i}\right) e^{y_{i}{ }^{2} / 2 \sigma_{n}{ }^{2}\left(4 \sigma_{n}{ }^{2}+1\right)}\right. \\
& \left.\quad \times\left[3 \sqrt{2 \pi} q y_{i} \sigma_{n}{ }^{4}\left(4 \sigma_{n}{ }^{2}+1\right)+\sqrt{2 \pi} q y_{i}{ }^{3} \sigma_{n}{ }^{2}\right]\right\}
\end{aligned}
$$

where $q=1 / \sigma_{n} \sqrt{4 \sigma_{n}^{2}+1}$.

Therefore, $f_{\mathrm{Nak}, 2}\left(y_{i} \mid H_{1}\right)$ can be written as

$$
\begin{aligned}
& f_{\mathrm{Nak}, 2}\left(y_{i} \mid H_{1}\right) \\
& =\frac{8 e^{-y_{i}^{2} / 2 \sigma_{n}{ }^{2}}}{\sqrt{2 \pi} \sigma_{n}\left(4 \sigma_{n}{ }^{2}+1\right)^{3}} \\
& \quad \times\left\{y_{i}^{2} \sigma_{n}{ }^{2}+2 \sigma_{n}{ }^{4}\left(4 \sigma_{n}{ }^{2}+1\right)+q \sqrt{2 \pi} e^{y_{i}{ }^{2} / 2 \sigma_{n}{ }^{2}\left(4 \sigma_{n}{ }^{2}+1\right)}\right. \\
& \left.\quad \times\left[P_{d 1, \mathrm{Nak}}-Q\left(q y_{i}\right)\right]\left[3 y_{i} \sigma_{n}{ }^{4}\left(4 \sigma_{n}{ }^{2}+1\right)+y_{i}{ }^{3} \sigma_{n}{ }^{2}\right]\right\} .
\end{aligned}
$$

A similar expression for $f\left(y_{i} \mid H_{0}\right)$ can be obtained by

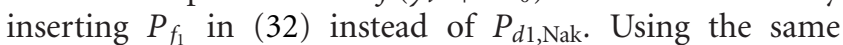


procedure as in Appendix B.2, in addition to making use of $[9,14.4-14]$ and the result in $[9,14.4-15]$, thus $P_{d 2, \mathrm{Nak}, 2}$ can be derived and given by

$$
\begin{aligned}
& P_{d 2, \mathrm{Nak}, 2} \\
& =\frac{1}{\left(4 \sigma_{n}{ }^{2}+1\right)^{3}} \\
& \quad \times\left[4 \sigma_{n}{ }^{4}+\frac{8 \sigma_{n}^{2}}{q}+\left(\frac{6 \sigma_{n}^{2}+1}{q^{3} \sigma_{n}{ }^{3}}\right) P_{d 1, \mathrm{Nak}, 2}\right. \\
& \left.\quad-\frac{3}{q^{2}}\left(\sqrt{4 \sigma_{n}{ }^{2}+1}-1\right)-\frac{1}{4 q^{3} \sigma_{n}{ }^{3}}(1-\varphi)^{2}(2+\varphi)\right]
\end{aligned}
$$

where $\varphi=1 / \sqrt{4 \sigma_{n}^{2}+1}$, and $P_{d 1, \mathrm{Nak}, 2}$ is obtained by substituting $m=2$ in (9).

$P_{f 2, \mathrm{Nak}, 2}$ can be obtained analogous to (33) while replacing $P_{d 1, N a k, 2}$ with $P_{f_{1}}$.

3.3. Overall Probabilities of False Alarm and Detection at the Fusion Center. Finally, the false alarm and detection probabilities in the fusion center can be expressed as a function of the probabilities at the output of the fading channels of the second stage (i.e., input to the fusion center) using the binomial expansion formula as studied before in [5, equations (1) and (2)] as follows:

$$
P_{F}=\sum_{i=t}^{K}\left(\begin{array}{c}
K \\
i
\end{array}\right) P_{f_{2}}^{i}\left(1-P_{f_{2}}\right)^{K-i},
$$

where $t$ is the threshold.

For the same $t$, the corresponding $P_{D}$ is

$$
P_{D}=\sum_{i=t}^{K}\left(\begin{array}{c}
K \\
i
\end{array}\right) P_{d 2, \mathrm{Nak}}^{i}\left(1-P_{d 2, \mathrm{Nak}}\right)^{K-i}
$$

\section{Diversity at the Local Sensors}

In this section, Square Law Combining (SLC) and Square Law Selection (SLS) Diversity schemes are employed in order to improve the energy detection performance at the local sensors. In Section 4.1 results for SLC are obtained, and in Section 4.2 results for SLS are depicted.

4.1. SLC Diversity. In this scheme, the outputs of the square law devices (square and integrate operation per diversity branch), denoted as $\left\{g_{i l}\right\}_{l=1}^{L}$, where $L$ is the number of diversity branches, are combined to yield a new decision statistic $g_{i, \text { SLC }}=\sum_{l=1}^{L} g_{i l}$. Under $H_{0}$ and for AWGN channels, adding $L$ i.i.d central chi-square variates, each with $\mathrm{N}$ DOFs and variance $\sigma^{2}$, will result in another chi-square variate with LN DOFs and the same variance $\sigma^{2}$.

Therefore, analogous to $(7), P_{f, \text { SLC }}$ can be written as $[8$, equation (10)]

$$
P_{f, \text { SLC }}=\frac{\Gamma\left(L N / 2, \lambda / 2 \sigma^{2}\right)}{\Gamma(L N / 2)} .
$$

Likewise, under $H_{1}, g_{i, \text { SLC }}$ will be a chi-square variate with LN DOFs, non-centrality parameter $\sum_{l=1}^{L} a \gamma_{l} \triangleq a \gamma_{t}$, and variance $\sigma^{2}$. Hence, $P_{d}$ at the combiner output for AWGN channels, $P_{d, \mathrm{SLC}}$, can be evaluated by analogy to $(8)$ as $[8$, equation (11)]

$$
P_{d, \mathrm{SLC}}=Q_{L N / 2}\left(\sqrt{\frac{a \gamma_{t}}{\sigma^{2}}}, \sqrt{\frac{\lambda}{\sigma^{2}}}\right) .
$$

In SLC, it is necessary to study both cases of i.i.d. and correlated diversity branches. In the following subsections, the probability of detection is averaged over both i.i.d and correlated Nakagami Channels.

4.1.1. Independent and Identically Distributed Nakagami Channels. The PDF of $\gamma_{t}$ for $L$ i.i.d. Nakagami branches is quite similar to that in (4), while replacing each $m$ by $m L$ and each $\bar{\gamma}$ by $L \bar{\gamma}$. Hence, $P_{d, \mathrm{Nak}, \mathrm{SLC}, \text { i.i.d }}$ is equivalent to $P_{d, \mathrm{Nak}}$ in (9) after replacing each $m$ by $L m$, each $\bar{\gamma}$ by $L \bar{\gamma}$, and each $N$ by $L N$.

4.1.2. Correlated Nakagami Channels. For L correlated Nakagami branches, the PDF of $\gamma_{t}$ is as given in [13, equation (18)]:

$$
\begin{aligned}
& P_{\gamma}(\gamma) \\
& =\frac{(\gamma m / \bar{\gamma})^{L m-1} e^{-\gamma m / \bar{\gamma}(1-\rho)} \mathfrak{B}}{(\bar{\gamma} / m)(1-\rho)^{m(L-1)}(1-\rho+L \rho)^{m} \Gamma(L m)},
\end{aligned}
$$

where $\mathfrak{B}$ denotes ${ }_{1} F_{1}(m, L m, L m \rho \gamma / \bar{\gamma}(1-\rho)(1-\rho+2 \rho))$, and $\rho$ is the correlation coefficient. By averaging $P_{d, \mathrm{SLC}}$, noise SLC, over (38), $P_{d 1, \text { Nak,SLC,corr }}$ can be obtained.

Results are obtained using Dual Diversity, that is, $L=$ 2 in (38), and using the same approach used to obtain the average probability of detection over Nakagami channels in Appendix A.1. Hence $P_{d 1, \mathrm{Nak}, \mathrm{SLC}, \text { corr }}$ is given by

$$
\begin{aligned}
& P_{d 1, \text { Nak,SLC,corr }} \\
& =\eta \sum_{j=0}^{\infty} \frac{\Gamma(\alpha+j) \Gamma(\beta) c^{j}}{\Gamma(\alpha) \Gamma(\beta+j) j !}\left(\frac{\sigma^{2}}{a}\right)^{j} \\
& \times\left[\frac{2^{n+j-1}(n+j-1) !}{v^{2(n+j)}\left(\frac{b_{1}{ }^{2}}{v^{2}+b_{1}{ }^{2}}\right) e^{-b_{2}{ }^{2} v^{2} / 2\left(v^{2}+b_{1}{ }^{2}\right)}}\right. \\
& \quad \times \sum_{k=0}^{n+j-1} \epsilon_{k}\left(\frac{v^{2}}{v^{2}+b_{1}{ }^{2}}\right)^{k} L_{k}\left(-\frac{b_{2}{ }^{2} b_{1}{ }^{2}}{2\left(v^{2}+b_{1}{ }^{2}\right)}\right) \\
& +\sum_{i=1}^{L N / 2-1} \frac{\Gamma((z+2 j+1) / 2)\left(b_{2}{ }^{2} / 2\right)^{i} e^{-b_{2}{ }^{2} / 2}}{2 i !\left(\left(v^{2}+b_{1}{ }^{2}\right) / 2\right)^{(z+2 j+1) / 2}} \\
& \left.\quad \times{ }_{1} F_{1}\left(\frac{z+2 j+1}{2}, i+1, \frac{b_{2}{ }^{2} b_{1}{ }^{2}}{2\left(v^{2}+b_{1}{ }^{2}\right)}\right)\right]
\end{aligned}
$$


where $c=2 m \rho / \bar{\gamma}(1-\rho)(1+\rho), \quad \eta=2\left(m \sigma^{2} / a \bar{\gamma}\right)^{2 m} / \Gamma(2 m)(1-$ $\rho)^{m}(1+\rho)^{m}, z=4 m-1, n=2 m, \alpha=m, \beta=2 m, v^{2}=$ $2 m \sigma^{2} / a \bar{\gamma}(1-\rho)$, and

$$
\epsilon_{k}= \begin{cases}1, & k<n+j-1, \\ 1+\frac{v^{2}}{b_{1}^{2}}, & k=n+j-1 .\end{cases}
$$

In the above equation, up to 40 terms are sufficient after which the probability of detection is insensitive to any increments to 3 significant digits independent on the values of false-alarm as shown in Table 1.

After taking 100 terms of (39) it was found that the maximum difference does not exceed $0.0445 \%$ as compared with results obtained by taking 40 terms for the parameters under consideration.

4.2. SLS Diversity. In the SLS diversity scheme, the branch with maximum decision statistic $g_{\text {SLS }}=\max \left(g_{1}, g_{2}, \ldots, g_{L}\right)$ is to be selected. Thus, $P_{f 1}$ for SLS, $P_{f 1 \text {,SLS }}$ can be written as in [8, equation (14)]:

$$
P_{f 1, \mathrm{SLS}}=1-\left(\frac{1-\Gamma\left(N / 2, \lambda / 2 \sigma^{2}\right)}{\Gamma(N / 2)}\right)^{L} .
$$

Using [8, equation (16)] $P_{d 1, \mathrm{SLS}, \mathrm{Nak}}$ for $L$ independent Nakagami branches is given by

$$
P_{d 1, \mathrm{SLS}, \mathrm{Nak}}=1-\prod_{i=1}^{L}\left(1-P_{d 1, \mathrm{Nak}}\left(\bar{\gamma}_{i}, N\right)\right),
$$

where $P_{d 1, \mathrm{Nak}}\left(\bar{\gamma}_{i}, N\right)$ is obtained as in (9).

\section{Numerical and Simulation Results}

The Receiver Operating Characteristics (ROC) [14], probability of detection $P_{D}$ versus $P_{F}$, for different situations of interest and different parameters such as the Nakagami fading parameter, the number of local sensors $(K)$, or SNR values are depicted. In all of the forthcoming results, the number of samples $N$ is taken equal to 4 . Figure 2 shows the performance of the system under consideration for different Nakagami fading parameters $(m=1 / 2,1,2)$ when $K=2$. For the sake of comparison with the noiseless second link channels, results for direct connection between the sensors and the fusion center are included. It is clear that fading deteriorates the performance. The degradation decreases with the increase of $m$, that is less fading depth. Better performance is achieved for higher SNR values $(\bar{\gamma}=20 \mathrm{~dB})$ as shown in Figure 3. Figure 4 depicts the performance to show the effect of increasing the number of sensors up to 4 . It is clear that the performance improves drastically with the increase of $K$. For further improvement diversity techniques are employed at the local sensors. Figure 5 displays the performance for dual SLC for different Nakagami fading assuming independent diversity branches. Similar results are depicted at Figure 6 for correlation coefficient equals 0.7. Both figures show that diversity appreciably improves the

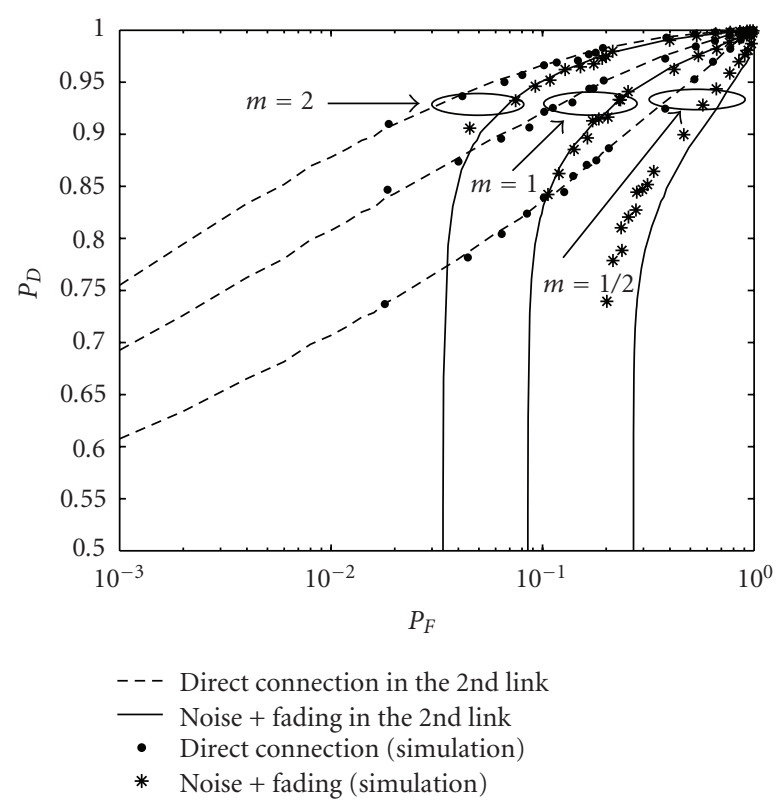

Figure 2: $P_{D}$ versus $P_{F}$ for $K=2$, for different Nakagami fading, $\bar{\gamma}=10 \mathrm{~dB}$.

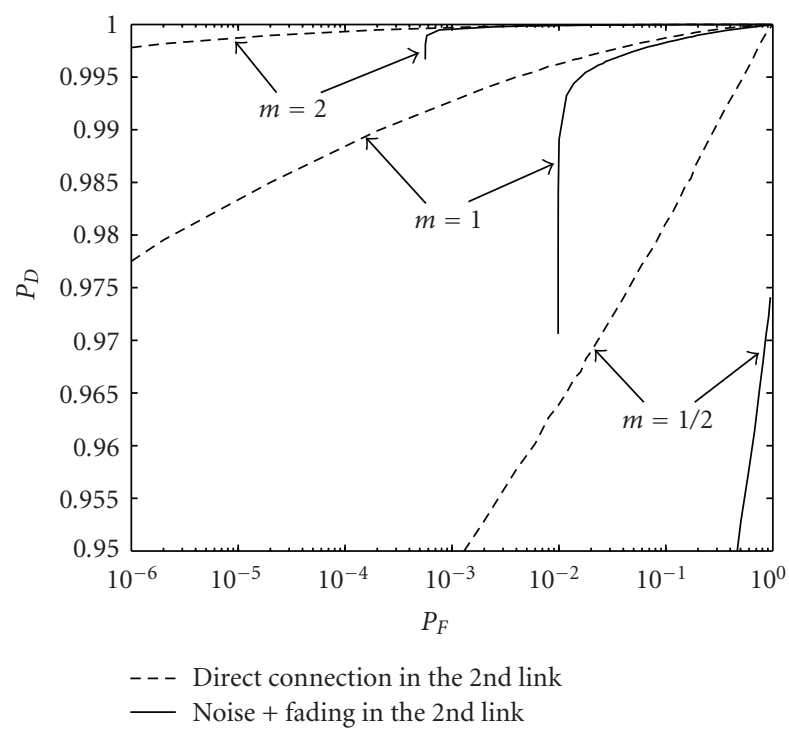

Figure 3: $P_{D}$ versus $P_{F}$ for $K=2$, for different Nakagami fading, $\bar{\gamma}=20 \mathrm{~dB}$.

performance and correlation degrades it as expected. Finally, Figure 7 represents results for an easier diversity system known as SLS for the dual situation. Although the performance is improved relative to the nondiversity situation, it is comparatively less than SLC system. Simulation results for Nakagmai fading are obtained using the technique described in [15]. Depicted results are shown in Figures 2 and 4. It is to be noted that simulation results coincide with those obtained theoretically with high degree of accuracy for all of the fading parameters under consideration. 
TABLE 1

\begin{tabular}{lcccc}
\hline No. of terms & $P_{f}=0.01$ & $P_{f}=0.05$ & $P_{f}=0.1$ & $P_{f}=0.5$ \\
\hline 40 & $P_{d 1, \mathrm{SLC}}=0.785$ & $P_{d 1, \mathrm{SLC}}=0.865$ & $P_{d 1, \mathrm{SLC}}=0.901$ & $P_{d 1, \mathrm{SLC}}=0.977$ \\
50 & $P_{d 1, \mathrm{SLC}}=0.785$ & $P_{d 1, \mathrm{SLC}}=0.865$ & $P_{d 1, \mathrm{SLC}}=0.901$ & $P_{d 1, \mathrm{SLC}}=0.977$ \\
60 & $P_{d 1, \mathrm{SLC}}=0.785$ & $P_{d 1, \mathrm{SLC}}=0.865$ & $P_{d 1, \mathrm{SLC}}=0.901$ & $P_{d 1, \mathrm{SLC}}=0.977$ \\
\hline
\end{tabular}

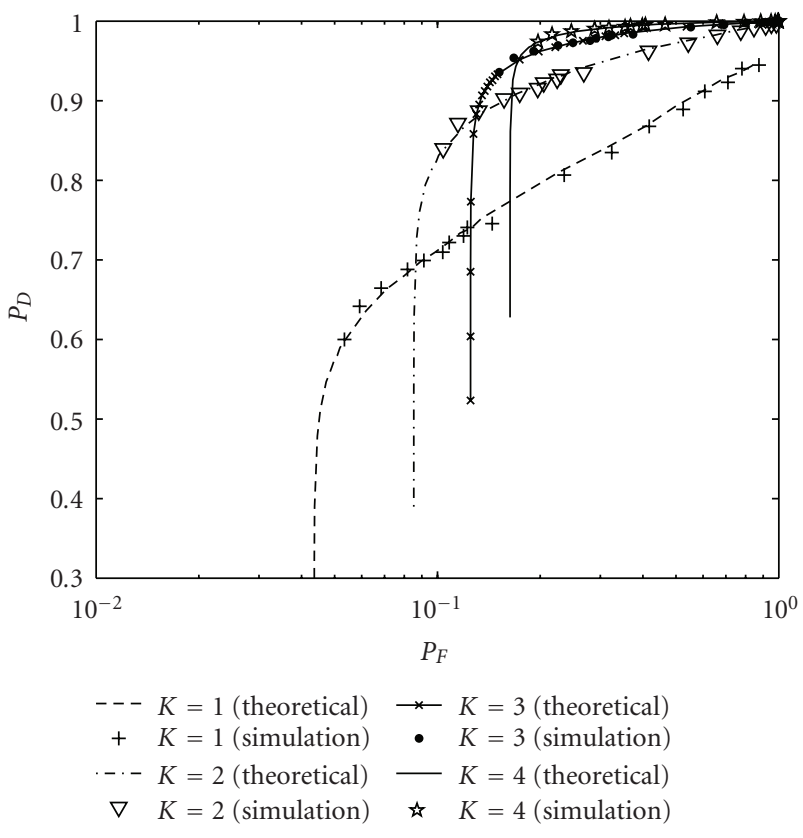

Figure 4: $P_{D}$ versus $P_{F}$ for different number of sensors assuming Rayleigh fading channels in both links, $\bar{\gamma}=10 \mathrm{~dB}$.

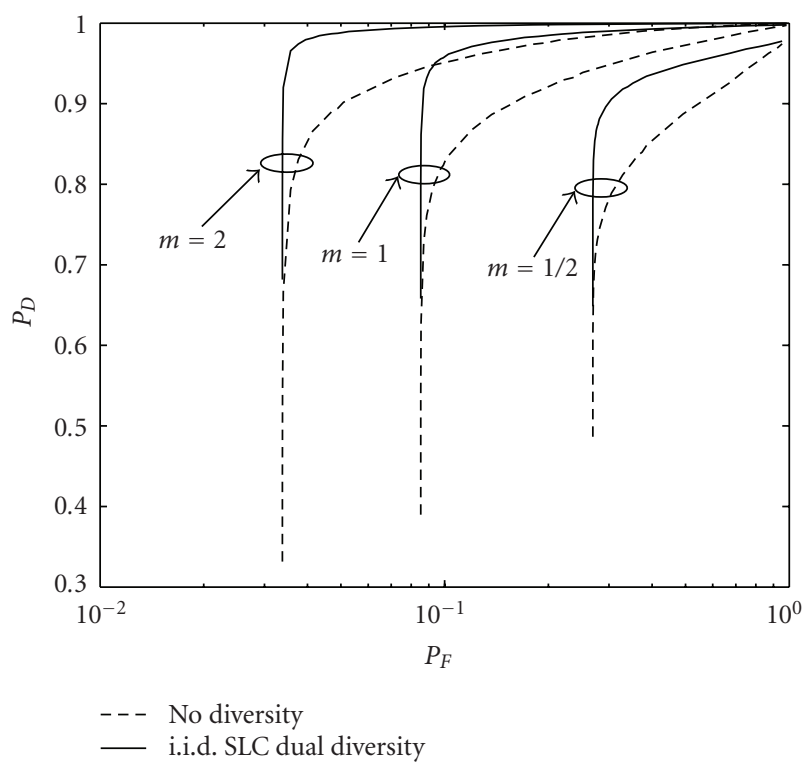

FIgure 5: $P_{D}$ versus $P_{F}$ for $K=2$ employing Dual SLC (i.i.d. diversity branches) for different Nakagami fading parameters, $\bar{\gamma}=$ $10 \mathrm{~dB}$.

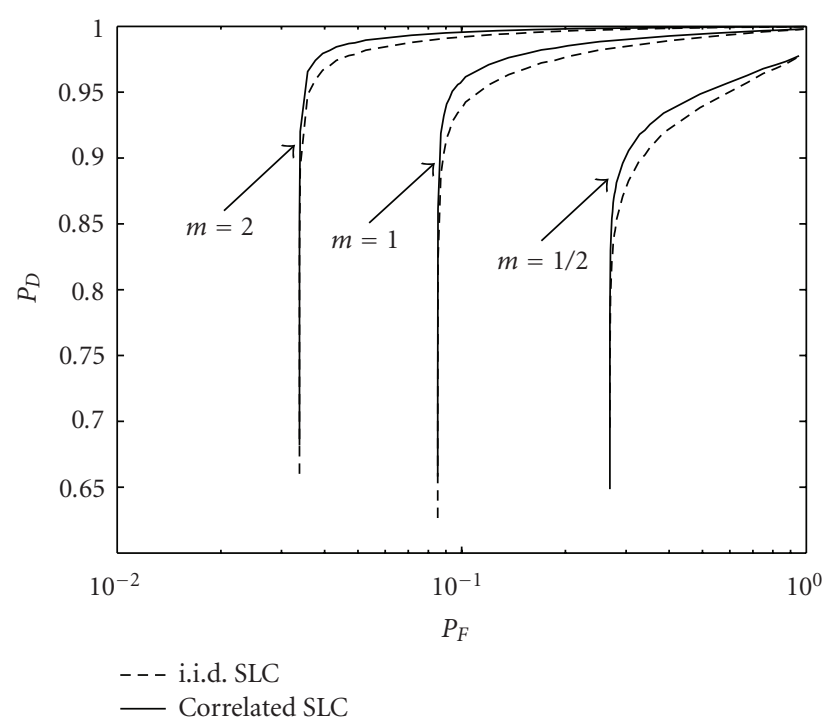

FIgure 6: $P_{D}$ versus $P_{F}$ for $K=2$ employing Dual SLC for both i.i.d. and correlated $(\rho=0.7)$ diversity branches, $\bar{\gamma}=10 \mathrm{~dB}$.

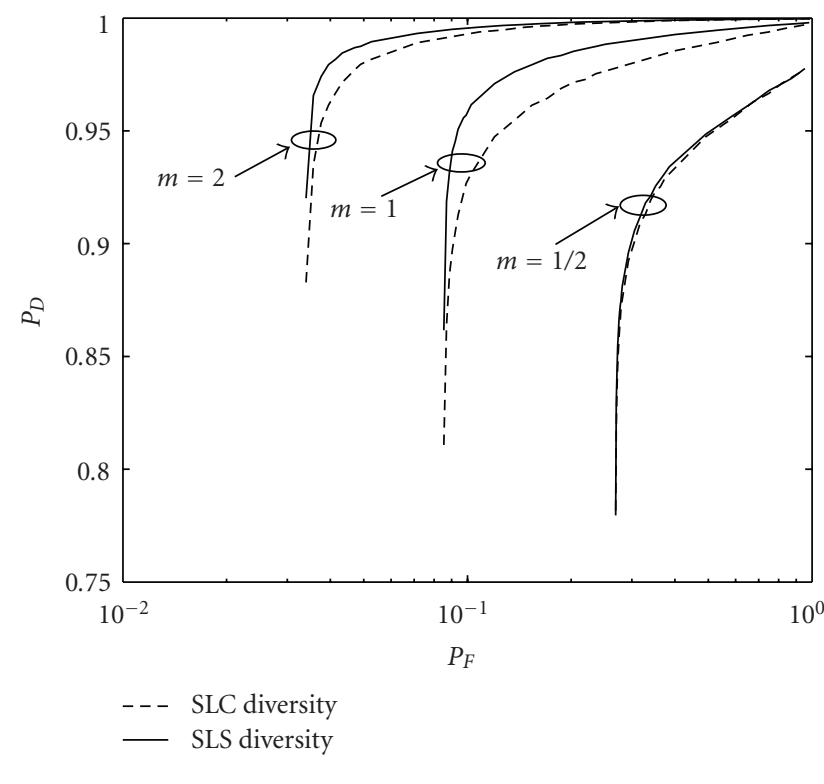

Figure 7: $P_{D}$ versus $P_{F}$ for $K=2$ employing both Dual SLS and Dual SLC (i.i.d. diversity branches) for different Nakagami fading parameters, $\bar{\gamma}=10 \mathrm{~dB}$. 


\section{Conclusion}

Comprehensive analytical and simulation results for decentralized detection of unknown ignals are included in this paper. AWGN and Nakagami fading are assumed in both channels. Dual SLC and SLS are employed at the local sensors for further improvement of the performance. Depicted results show that the performance improves with the decrease of the fading depth, that is, increase of the Nakagmi parameter " $m$ " and increase of the number of local sensors.

\section{Appendices}

\section{A. Analytical Results for the Probabilities of False Alarm and Detection at the Local Sensors}

A.1. Evaluation of Probability of Detection over Nakagami Fading Channels. Given that

$$
\begin{aligned}
& P_{d 1, \mathrm{Nak}}=\int_{0}^{\infty} f_{\mathrm{Nak}}(\gamma) Q_{N / 2}\left(\sqrt{\frac{a \gamma}{\sigma^{2}}}, \sqrt{\frac{\lambda}{\sigma^{2}}}\right) d \gamma, \\
& P_{d 1, \mathrm{Nak}}=\zeta \int_{0}^{\infty} x^{u} \exp \left(-\frac{v^{2} x^{2}}{2}\right) Q_{M}\left(b_{1} x, b_{2}\right) d x=\zeta G_{M},
\end{aligned}
$$

where $\zeta=2 / \Gamma(m)\left(m \sigma^{2} / a \bar{\gamma}\right)^{m}, u=2 m-1, v^{2}=$ $2 m \sigma^{2} / a \bar{\gamma}, M=N / 2, b_{1}=1$, and $b_{2}=\sqrt{\lambda / \sigma^{2}}$.

Using the recursive expansion of $Q_{M}$ function given by $[10,4.61]$

$$
\begin{aligned}
G_{M}= & \int_{0}^{\infty} x^{u} \exp \left(-\frac{v^{2} x^{2}}{2}\right) Q_{M-1}\left(b_{1} x, b_{2}\right) d x \\
& +\int_{0}^{\infty} x^{u} \exp \left(-\frac{v^{2} x^{2}}{2}\right)\left(\frac{b_{2}}{b_{1} x}\right)^{M-1} \\
& \quad \times \exp \left(-\frac{\left(b_{1}{ }^{2} x^{2}+b_{2}^{2}\right)}{2}\right) I_{M-1}\left(b_{1} b_{2} x\right) d x
\end{aligned}
$$

$G_{M}$ can be recursively evaluated as follows

$$
\begin{aligned}
G_{M}= & G_{M-1}+J_{M-1} \\
= & G_{M-2}+J_{M-2}+J_{M-1} \\
& \vdots \\
= & G_{1}+J_{1}+J_{2}+\cdots+J_{M-2}+J_{M-1} \\
= & G_{1}+\sum_{i=1}^{M-1} J_{i} .
\end{aligned}
$$

With the aid of $[11,6.631]$ and $\left[16\right.$, equation (29)], $J_{i}$ is given by

$$
\begin{aligned}
J_{i}= & \frac{\Gamma((u+1) / 2)\left(b_{2}{ }^{2} / 2\right)^{i} e^{-b_{2}{ }^{2} / 2}}{2(i !)\left(\left(v^{2}+b_{1}{ }^{2}\right) / 2\right)^{(u+1) / 2}} \\
& \times{ }_{1} F_{1}\left(\frac{u+1}{2} ; i+1 ; \frac{b_{1}{ }^{2} b_{2}{ }^{2}}{2\left(v^{2}+b_{1}{ }^{2}\right)}\right) .
\end{aligned}
$$

$G_{1}$, for integer $m$, can be found with the aid of [16, equation (25)], while for the case of $m=1 / 2, G_{1}$ is obtained using [ 17 , equation (63)].

\section{B. Analytical Results for the Probabilities of False Alarm and Detection at the Fusion Center}

B.1. Evaluation of $f_{N a k, 1 / 2}\left(y_{i} \mid u_{i}\right)$. Setting $m=1 / 2$ in (20) yields

$$
\begin{aligned}
& f_{\mathrm{Nak}, 1 / 2}\left(y_{i} \mid u_{i}=1\right) \\
& =\frac{\sqrt{2}}{\Gamma(1 / 2) \sqrt{2 \pi} \sigma_{n}} e^{-y^{2} / 2\left(\sigma_{n}{ }^{2}+1\right)} \int_{0}^{\infty} e^{-\left(x-\left(y /\left(\sigma_{n}{ }^{2}+1\right)\right)\right)^{2} / 2\left(\sigma_{n}{ }^{2} /\left(\sigma_{n}{ }^{2}+1\right)\right)} d x \\
& =\frac{\sqrt{2}}{\Gamma(1 / 2) \sqrt{\sigma_{n}{ }^{2}+1}} e^{-y^{2} / 2\left(\sigma_{n}{ }^{2}+1\right)} Q\left(\frac{-y}{\sigma_{n} \sqrt{\sigma_{n}^{2}+1}}\right) .
\end{aligned}
$$

B.2. Evaluation of the Probability of Detection $\left(P_{d_{2}}\right)$ at the Output of the Nakagami Fading Channel of the Second Stage for the Case of $m=1 / 2$. Having

$$
\begin{aligned}
P_{d 2, \mathrm{Nak}, 1 / 2}= & \int_{0}^{\infty} \frac{\sqrt{2}}{\Gamma(1 / 2)} \sigma_{n} w e^{-y^{2} / 2\left(\sigma_{n}{ }^{2}+1\right)} \\
& \times\left[P_{d 1, \mathrm{Nak}, 1 / 2}+\left(1-2 P_{d 1, \mathrm{Nak}, 1 / 2}\right) Q(w y)\right] d y
\end{aligned}
$$

where $w=1 / \sigma_{n} \sqrt{\sigma_{n}^{2}+1}$

$$
\begin{aligned}
P_{d 2, \mathrm{Nak}, 1 / 2} & =\frac{\sqrt{2}}{\Gamma(1 / 2)} \sigma w\left[A P_{d 1, \mathrm{Nak}, 1 / 2}+\left(1-P_{d 1, \mathrm{Nak}, 1 / 2}\right) B\right] \\
A & =\int_{0}^{\infty} e^{-y^{2} / 2\left(\sigma_{n}{ }^{2}+1\right)} d y=\frac{\sqrt{2 \pi\left(\sigma_{n}^{2}+1\right)}}{2} \\
B & =\int_{0}^{\infty} Q(w y) e^{-y^{2} / 2\left(\sigma_{n}{ }^{2}+1\right)} d y .
\end{aligned}
$$

Making use of the approximate expansion for $Q(x)$ as presented in $[18$, equation (6)]:

$$
B=\frac{1}{w \sqrt{2 \pi}} \int_{0}^{\infty} \frac{1}{\sqrt{1+x^{2}}} e^{-\left(x^{2} / 2\right)\left(1+\sigma_{n}{ }^{2}\right)} d x
$$


By making the change of variables, $z=x^{2}$, and using [11, 3.364], thus

$$
B=\frac{1}{2 w \sqrt{2 \pi}} e^{\left(1+\sigma_{n}{ }^{2}\right) / 4} K_{0}\left(\frac{1+\sigma_{n}^{2}}{4}\right) .
$$

Similarly, $P_{f 2, \mathrm{Nak}, 1 / 2}$ can be justified following the same steps.

B.3. Evaluation of $f_{\text {Ray }}\left(y_{i} \mid u_{i}=1\right)$. Following the same analysis as in B.2, while setting $m=1$ in (20) yields

$$
\begin{aligned}
& f_{\text {Ray }}\left(y_{i} \mid u_{i}=1\right) \\
& =\frac{2}{\sqrt{2 \pi} \sigma_{n}} e^{-y^{2} / 2\left(\sigma_{n}{ }^{2}+1\right)} \int_{0}^{\infty} x e^{-\left(x-y /\left(\sigma_{n}{ }^{2}+1\right)\right)^{2} /\left(2 \sigma_{n}{ }^{2} /\left(\sigma_{n}{ }^{2}+1\right)\right)} d x \\
& =\frac{2 \sigma_{n}}{\sqrt{2 \pi}\left(1+2 \sigma_{n}^{2}\right)} e^{-y^{2} / 2 \sigma_{n}^{2}}\left[1+\sqrt{2 \pi} p y e^{(p y)^{2} / 2} Q(-p y)\right] \text {, }
\end{aligned}
$$

where $p=1 / \sigma_{n} \sqrt{2 \sigma_{n}^{2}+1}$.

B.4. Evaluation of $f_{\mathrm{Nak,2}}\left(y_{i} \mid u_{i}=1\right)$. Having

$$
\begin{aligned}
& f_{\mathrm{Nak}, 2}\left(y_{i} \mid u_{i}=1\right) \\
& =\frac{8}{\sqrt{2 \pi} \sigma_{n}} e^{-2 y^{2} /\left(4 \sigma_{n}^{2}+1\right)} \\
& \quad \times \int_{-y /\left(4 \sigma_{n}^{2}+1\right)}^{\infty}\left(t+\frac{y}{4 \sigma_{n}^{2}+1}\right)^{3} e^{-t^{2} / 2\left(\sigma_{n}{ }^{2} /\left(4 \sigma_{n}{ }^{2}+1\right)\right)} d t \\
& =\frac{8}{\sqrt{2 \pi} \sigma_{n}} e^{-2 y^{2} /\left(4 \sigma_{n}{ }^{2}+1\right)}[X+Y+W+Z],
\end{aligned}
$$

where

$$
\begin{aligned}
X= & e^{-y^{2} / 2 \sigma_{n}^{2}\left(4 \sigma_{n}^{2}+1\right)}\left[\frac{y^{2}{\sigma_{n}}^{2}}{\left(4 \sigma_{n}^{2}+1\right)^{3}}+\frac{2 \sigma_{n}^{4}}{\left(4 \sigma_{n}^{2}+1\right)^{2}}\right], \\
Y= & -\frac{3 y^{2} \sigma_{n}^{2}}{\left(4 \sigma_{n}^{2}+1\right)^{3}}+3 \sqrt{2 \pi} \frac{y \sigma_{n}^{3}}{\left(4 \sigma_{n}^{2}+1\right)^{2} \sqrt{4 \sigma_{n}^{2}+1}} \\
& \times Q\left(-\frac{y}{\sigma_{n} \sqrt{4 \sigma_{n}^{2}+1}}\right) \\
W= & \frac{3 y^{2} \sigma_{n}{ }^{2}}{\left(4 \sigma_{n}{ }^{2}+1\right)^{3}} e^{-y^{2} / 2 \sigma_{n}{ }^{2}\left(4 \sigma_{n}{ }^{2}+1\right)} .
\end{aligned}
$$

Also,

$$
Z=\sqrt{2 \pi}\left(\frac{y}{4 \sigma_{n}^{2}+1}\right)^{3} \frac{\sigma_{n}}{\sqrt{4 \sigma_{n}^{2}+1}} Q\left(-\frac{y}{\sigma_{n} \sqrt{4 \sigma_{n}^{2}+1}}\right) .
$$

Finally,

$$
\begin{aligned}
& f_{\mathrm{Nak}, 2}\left(y_{i} \mid u_{i}=1\right) \\
& =\frac{8}{\sqrt{2 \pi} \sigma_{n}\left(4 \sigma_{n}{ }^{2}+1\right)^{3}} e^{-y^{2} / 2 \sigma_{n}{ }^{2}} \\
& \quad \times\left\{y^{2}{\sigma_{n}}^{2}+2{\sigma_{n}}^{4}\left(4 \sigma_{n}{ }^{2}+1\right)+Q(-q y) e^{y^{2} / 2 \sigma_{n}{ }^{2}\left(4 \sigma_{n}{ }^{2}+1\right)}\right. \\
& \left.\quad \times\left[3 \sqrt{2 \pi} q y{\sigma_{n}}^{4}\left(4 \sigma_{n}{ }^{2}+1\right)+\sqrt{2 \pi} q y^{3}{\sigma_{n}}^{2}\right]\right\} .
\end{aligned}
$$

\section{References}

[1] R. Viswanathan and P. K. Varshney, "Distributed detection with multiple sensors: part I-fundamentals," Proceedings of the IEEE, vol. 85, no. 1, pp. 54-63, 1997.

[2] R. S. Blum, S. A. Kassam, and H. V. Poor, "Distributed detection with multiple sensors: part II-advanced topics," Proceedings of the IEEE, vol. 85, no. 1, pp. 64-79, 1997.

[3] Z. Chair and P. K. Varshney, "Optimal data fusion in multiple sensor detection systems," IEEE Transactions on Aerospace and Electronic Systems, vol. 22, no. 1, pp. 98-101, 1986.

[4] C. Lee and J. Chao, "Optimum local decision space partitioning for distributed detection," IEEE Transactions on Aerospace and Electronic Systems, vol. 25, no. 4, pp. 536-544, 1989.

[5] E. K. Al-Hussaini and Y. A. El-Far, "Decentralized nonparametric detectors," IEEE Signal Processing Letters, vol. 4, no. 5, pp. 128-131, 1997.

[6] R. Niu, B. Chen, and P. K. Varshney, "Decision fusion rules in wireless sensor networks using fading channel statistics," in Proceedings of the 37th Annual Conference on Information Sciences and Systems (CISS '03), Baltimore, Md, USA, March 2003.

[7] H. Urkowitz, "Energy detection of unknown deterministic signals," Proceedings of the IEEE, vol. 55, no. 4, pp. 523-531, 1967.

[8] F. F. Digham, M.-S. Alouini, and M. K. Simon, "On the energy detection of unknown signals over fading channels," IEEE Transactions on Communications, vol. 55, no. 1, pp. 21-24, 2007.

[9] J. G. Proakis, Digital Communications, McGraw-Hill, New York, NY, USA, 2001.

[10] M. K. Simon and M.-S. Alouini, Digital Communication Over Fading Channels, John Wiley \& Sons, New York, NY, USA, 2nd edition, 2005.

[11] I. S. Gradshteyn and I. M. Ryzhik, Table of Integrals, Series and Products, Academic Press, Boston, Mass, USA, 4th edition, 2007.

[12] P. Dharmawansa, N. Rajatheva, and K. Ahmed, "On the distribution of the sum of Nakagami-m random variables," IEEE Transactions on Communications, vol. 55, no. 7, pp. 14071416, 2007.

[13] V. A. Aalo, "Performance of maximal-ratio diversity systems in a correlated Nakagami-fading environment," IEEE Transactions on Communications, vol. 43, no. 8, pp. 2360-2369, 1995.

[14] H. L. Vantrees, Detection, Estimation, and Modulation Theory, Part I, John Wiley \& Sons, New York, NY, USA, 2001.

[15] Q. T. Zhang, "Decomposition technique for efficient generation of correlated Nakagami fading channels," IEEE Journal on Selected Areas in Communications, vol. 18, no. 11, pp. 23852392, 2000.

[16] A. H. Nuttal, "Some integrals involving the $\mathrm{Q}_{\mathrm{M}}$-function," Tech. Rep., Naval Underwater Systems Center (NUSC), Washington, DC, USA, May 1974.

[17] A. H. Nuttal, "Some integrals involving the $Q$-function,” Tech. Rep., Naval Underwater Systems Center (NUSC), Washington, DC, USA, April 1972.

[18] Y. Chen and N. C. Beaulieu, "A simple polynomial approximation to the Gaussian Q-function and its application," IEEE Communications Letters, vol. 13, no. 2, pp. 124-126, 2009. 